\title{
Cytosolic chaperonin CCT possesses GTPase activity
}

\author{
Susumu Noguchi ${ }^{1}$, Kazuyoshi Toyoshima ${ }^{1}$, Soh Yamamoto ${ }^{1}$, Toshio Miyazaki ${ }^{1}$, Michiro Otaka ${ }^{2}$, \\ Sumio Watanabe ${ }^{2}$, Katsunori Imai ${ }^{3}$, Haruki Senoo ${ }^{3}$, Ryoji Kobayashi ${ }^{4}$, Mitsutoshi Jikei $^{5}$, Yasushi Kawata ${ }^{6}$, \\ Hiroshi Kubota ${ }^{1}$, Hideaki Itoh ${ }^{1, *}$

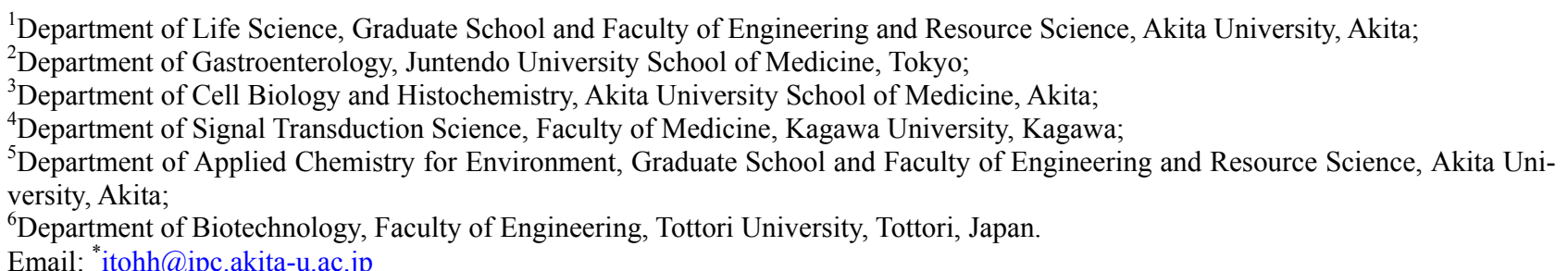

Received 30 July 2011; revised 11 August 2011; accepted 20 August 2011.

\begin{abstract}
Cytosolic chaperonin CCT (also known as TRiC) is a hetero-oligomeric cage-like molecular chaperone that assists in protein folding by ATPase cycle-dependent conformational changes. However, role of the nucleotide binding and hydrolysis in CCT-assisted protein folding is still poorly understood. We purified CCT by using ATP-Sepharose and other columns, and found that CCT possesses ability to hydrolyze GTP, with an activity level very similar to the ATPase activity. CCT was more resistant to proteinase $\mathrm{K}$ treatment in the presence of GTP or ATP. These results suggest that the GTPase activity of CCT may play a role in chaperone-assisted protein folding.
\end{abstract}

Keywords: Chaperonin; Molecular Chaperone; Protein Folding, Gtp

\section{INTRODUCTION}

Many cellular proteins require assistance with molecular chaperones to obtain functional conformations $[1,2]$. Molecular chaperones are also required for refolding of proteins unfolded or misfolded under stress condition including heat shock. Chaperonins are large cylindrical complexes that enhance the efficiency of protein folding [3]. These complexes are two-ring assemblies that posses a central cavity to accommodate non-native proteins, and the substrate proteins are released after completion of folding. The reaction cycle consists of a nucleotide-regulated conformational alteration between an open substrate-acceptor state and a closed folding-active state.

Chaperonins has been divided into two classes: group I chaperonins are found in prokaryotes and eukaryotic organelles including mitochondria and cytoplasm [4-6] whereas group II chaperonins are found in the eukaryotic cytosol and archaea [7,8]. Group I chaperonins, includeing E. coli GroEL and mitochondrial HSP60, consist of 14 subunits and require ring-shaped cofactor GroES for their chaperone activities [3,9]. Group II chaperonins, including cytosolic chaperonin CCT (also known as TRiC) of eukaryotes and archaeal chaperonins, share the double-ring structure with the group I members. However, the group II chapronins have several characters distinct from the group I chaperonins: the former consists of 16 or 18 subunits and uses a built-in lid called helical protrusion to close the cylindrical structure $[8,10]$.

CCT assists in folding of proteins in eukaryotic cytosol and has eight different subunits [8,11]. All subunits have conserved ATPase domain and divergent substrate binding domain [12]. CCT plays an essential role in folding a subset of cytosolic proteins. These substrates include actin, tubulin, WD40 domain $\beta$-propeller proteins and other proteins [13-15]. A critical question in understanding the mechanism of CCT-assisted protein folding is how ATP binding and hydrolysis drives the conformational change that stimulates productive folding. Although CCT has some features common to that of the type I chaperonin GroEL and roles of nucleotide binding and hydrolysis is well documented, CCT lacks the concerted action with co-chaperonin like GroES and uses built-in lid [16,17]. The existence of eight different subunits suggests that degree of ATPase activity may differ between subunit species [18,19]. However, mechanisms for controlling ATP binding and hydrolysis by CCT is still poorly understood.

Here, we purified CCT from porcine testis by a simple method, and found that CCT possesses a GTPase activ- 
ity in addition to an ATPase activity. We discuss roles of different nucleotides on CCT-assisted protein folding.

\section{MATERIALS AND METHODS}

\subsection{Antibodies}

Rat anti-CCT $\alpha$ antibody (Stressgen, BC, Canada) and alkaline phosphatase-conjugated anti-rat $\operatorname{IgG}$ (Chemicon International, CA, USA) were obtained from the source shown.

\subsection{Purification of CCT}

Large scale purification of CCT was performed as follows (see supplemental Figure 1). Porcine testis (300 g) was homogenized in $900 \mathrm{ml}$ buffer A $(250 \mathrm{mM}$ sucrose and $10 \mathrm{mM}$ Tris- $\mathrm{HCl} \mathrm{pH} 7.4)$. Soluble fraction was recovered after centrifugation $\left(20,000 \times \mathrm{g}, 15 \mathrm{~min}, 4^{\circ} \mathrm{C}\right)$ and ammonium sulfate was added to a concentration of $209 \mathrm{~g} / \mathrm{l}$. Supernatant was collected after centrifugation $(20,000 \times \mathrm{g}, 15 \mathrm{~min})$ and ammonium sulfate was added $(63 \mathrm{~g} / \mathrm{l})$ again. Precipitates were collected after centrifugation and dissolved in buffer A. Proteins were dialyzed overnight against buffer $\mathrm{A}$ and applied to a $50 \mathrm{ml}$ diethylaminoethyl (DEAE) cellulose (Whatman DE52, GE Healthcare, Amersham Place, UK) column. Pass-through fractions were collected and applied to a $10 \mathrm{ml}$ heparin-Sepharose (GE Healthcare) column in buffer A. Proteins were eluted with a linear gradient of $150-800 \mathrm{mM}$ $\mathrm{NaCl}$ in buffer A. Fractions containing CCT were applied to a $10 \mathrm{ml}$ ATP-Sepharose (Sigma, St Louis, MO, USA) column using buffer A supplemented with $5 \mathrm{mM}$ $\mathrm{MgCl}_{2}$ and $1 \mathrm{mM} \mathrm{CaCl}$, and proteins were eluted with buffer A supplemented with $5 \mathrm{mM}$ ATP and $10 \mathrm{mM}$ EDTA. Fractions containing CCT was applied to a $10 \mathrm{ml}$ Q-Sepharose (GE Healthcare) column in buffer A, and proteins were eluted with a linear gradient of $50-800$ $\mathrm{mM} \mathrm{NaCl}$ in buffer A. For small scale purification, the DEAE cellulose column was replaced by a Q-Sepharose column and proteins were purified from pass through fraction (P-fraction) and separatly from elute fraction (E-fraction) (supplementary Figure 2). Purified proteins were analyzed by SDS-PAGE [20] followed by immunoblotting with an antibody against CCT $\alpha$ [21], or two-dimensional gel electrophoresis (isoelectric focusing at $\mathrm{pH}$ 6.0-8.0 followed by SDS-PAGE) according to O'Farrell [22].

\subsection{Nucleotide hydrolysis analysis}

CCT $(0.05 \mu \mathrm{M})$ was incubated with $1 \mathrm{mM}$ ATP or GTP in buffer $\mathrm{D}(5 \mathrm{mM} \mathrm{MgCl} 2,100 \mathrm{mM} \mathrm{KCl}$ and $25 \mathrm{mM}$ HEPES-NaOH pH 7.4) at $37^{\circ} \mathrm{C}$, and concentration of free phosphate was determined by a method utilizing Malachite green as indicator [23] using Biomol Green Reagent (Biomol, Plymouth Meeting, PA, USA).
For HPLC analysis, purified CCT $(0.14 \mu \mathrm{M})$ was incubated with nucleotide (final concentration of $50 \mu \mathrm{M}$ ) at $37^{\circ} \mathrm{C}$. Nucleotides were separated on $\mathrm{C}_{18}$ reverse phase column (Mightysil PR-18CP, 4.6 mm ID x 150 mm, Kanto Kagaku: Tokyo, Japan) chromatography using HPLC $[4,5]$. The liquid chromatographic equipment consisted of a PU-1580 intelligent HPLC pump, LG-1580-02 ternary gradient unit (Jasco: Tokyo, Japan), SPD6A spectrophotometric detector, and CTO6A column oven (Shimadzu, Kyoto, Japan). Data were recorded and analyzed with a LabVIEW software system Version 7.1 (National Instruments: TX, U.S.A.). Chromatographic determination was performed at a flow rate of $1 \mathrm{ml} / \mathrm{min}$ at $37^{\circ} \mathrm{C}$, and the detection wavelength was set at $256 \mathrm{~nm}$.

\subsection{Protease Resistance Test}

Purified CCT was incubated with proteinase $\mathrm{K}$ as described previously [15] with following modifications. Nucleotides (5 mM ATP or GTP) and metal ions ( $5 \mathrm{mM}$ $\mathrm{MgCl}_{2}$ or $\mathrm{CaCl}_{2}$ ) were added to samples in buffer A. Proteinase $\mathrm{K}$ was added to the all samples at a final concentration of $20 \mu \mathrm{g} / \mathrm{ml}$. After incubation at $25^{\circ} \mathrm{C}$ for $5 \mathrm{~min}$, samples were analyzed by SDS-PAGE.

\subsection{Electron Microscopy}

Purified proteins were adsorbed onto collodion membranes (Nissin EM, Tokyo, Japan) and stained with 4\% uranyl acetate by a double carbon technique modified from the method of Lake [24]. Observation of samples was performed by a $\mathrm{H} 7650$ electron microscope (Hitachi, Tokyo, Japan).

\section{RESULTS}

In the present study, we first tested two different methods to purify CCT from porcine testis in small scale (supplementary Figure 2). One uses path through fraction (P-fraction) of Q-sepharose column and further purification using ATP-Sepharose and mono-Q columns. The other uses elute fraction (E-fraction) of the same Q-sepharose column and further purification using heparin-Sepharose column. Purified proteins were analyzed by SDS/PAGE followed by Coomassie Brilliant Blue staining or immunoblotting using an antibody against CCT (Figure 1). Several bands of approximately $60 \mathrm{kDa}$ were detected for the protein sample purified from P-fraction (Figure 1(a)) and one of these bands was recognized by an anti-CCT $\alpha$ antibody (Figure 1(b)). These observations are typical of the CCT complex previously described. On the contrary, a single protein band with a molecular mass of $60 \mathrm{kDa}$ was detected from the protein purified from E-fraction (Figure 1(c)). By immunoblotting, the single band was recognized by the 


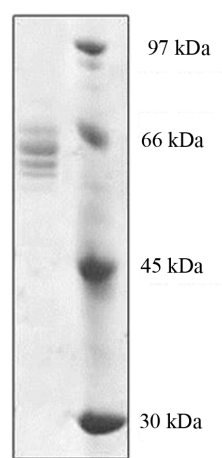

(a)

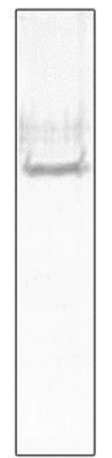

(b)

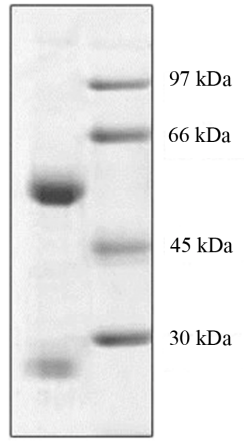

(c)

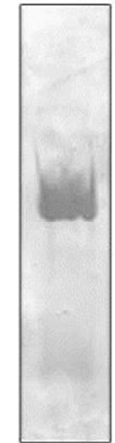

(d)
Figure 1. Gel electrophoresis analysis of CCT purified by two different methods. CCT purified from P-fraction (see supplemental Figure 2), (a) and (b) or from E-fraction (c) and (d) were analyzed by SDS-PAGE ( $9 \%$ polyacrylamide gel) followed by staining with Coomassie Brilliant Blue; (a) and (c) or immunoblotting using an anti CCT-alpha antibody (b) and (d).

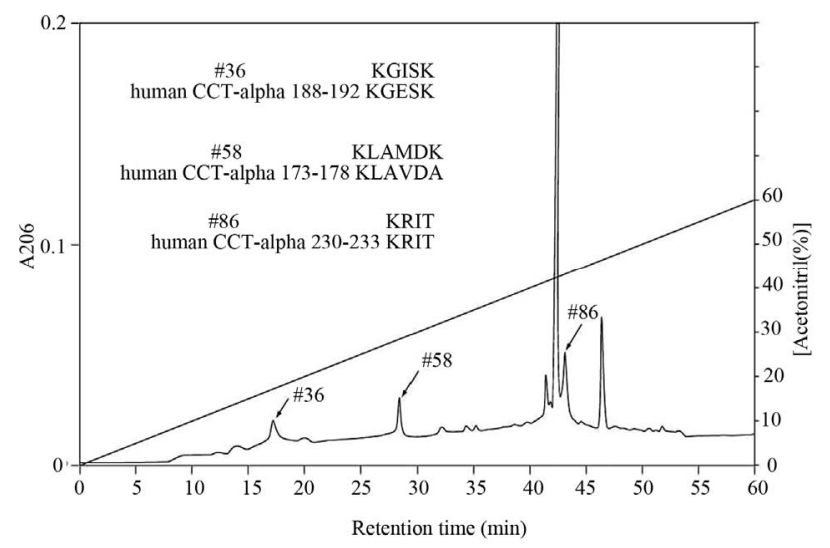

Figure 2. Amino acid sequences of lysyl endopeptidase digests of CCT purified by Heparin-Sepharose column chromatography.Lysyl endopeptidase digests of CCT purified from E-fraction were separated by reverse phase column chromatography. Peptide number 36, 58 and 8 were sequenced and compared with the sequence of human $\mathrm{CCT} \alpha$.

anti-CCT $\alpha$ antibody (Figure 1(d)). Amino acid sequencing of the protein purified from E-fraction indicated that a peptide fragment (no. 86) has an amino-acid sequence identical to that of the human CCT (Figure 2). In addition, amino-acid sequences of peptide no. 36 and 58 were similar to that of the human CCT. These results strongly suggest that major constituent of the protein complex purified from E-fraction is CCT $\alpha$. Analysis of the subunit composition by two-dimensional gel electrophoresis indicated that the sample purified by ATPagarose shows eight protein spots typical of CCT subunits (Figure 3(a)). In contrast, one or two protein spots were detected for the protein purified from E-fraction (Figure 3(b)). Gel filtration and native-PAGE analyses indicated that the protein purified from $\mathrm{P}$-fraction has a relative molecular mass of about $900 \mathrm{kDa}$, consistent with known molecular size of the CCT complex (Figure 4). In contrast, CCT purified from E-fraction was approximately $400 \mathrm{kDa}$. By electron microscopic analysis, typical ring-like structure was detected from the sample purified from P-fraction (Figure 5). In contrast, no ring-like structure was observed from the sample purified from E-fraction (data not shown). Based on these observations, we concluded that the protein purified from P-fraction was the complete CCT complex composed of eights different subunits, although the protein purified from E-fraction may be a partial CCT complex mainly composed of CCT $\alpha$.

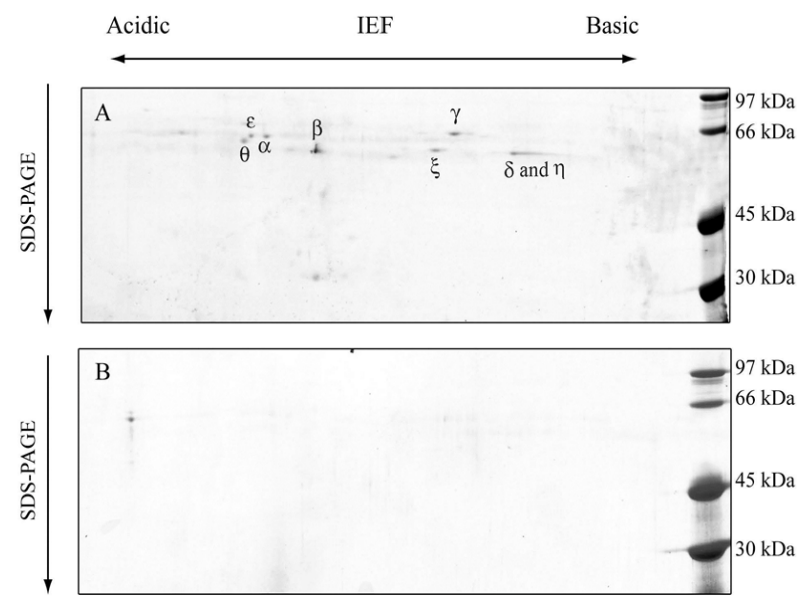

Figure 3. Two dimensional gel electrophoresis of purified CCT samples. CCT samples purified from P-fraction (a) or E-fraction (b) were analyzed by two-dimensional gel electorophoresis followed by staining with Coomassie Brilliant Blue.

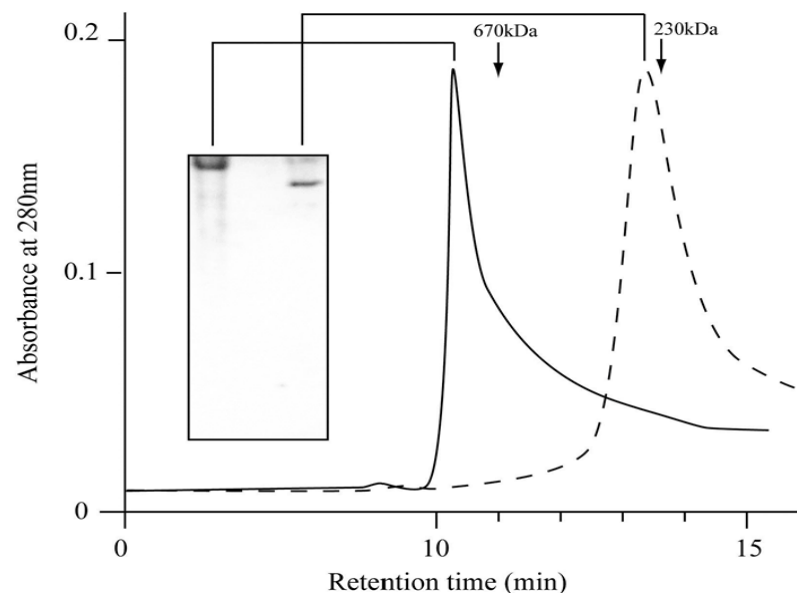

Figure 4. Gel filtration analysis of the purified CCT. CCT samples purified from P-fraction or E-fraction were separated on a TSK G4000SW column. CCT samples purified from P-fraction (solid line) or E-fraction (dashed line) were load on the gel filtration column, and elution profiles are shown. Data of native PAGE analysis are shown with the elution profiles. 


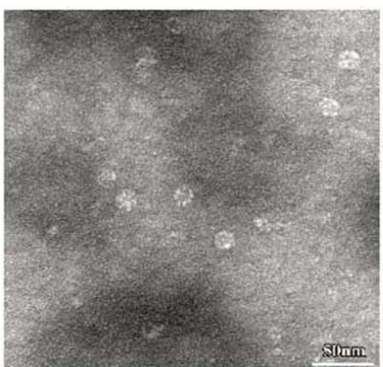

(a)

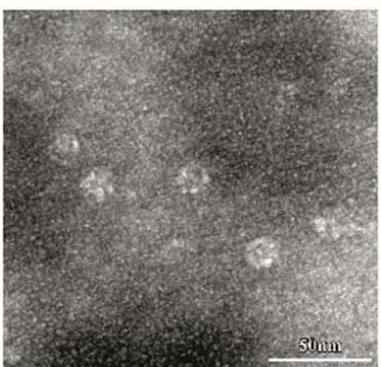

(b)
Figure 5. Electron microscopic analysis of purified CCT. For transmission electron microscopy, samples of CCT purified by ATP-Sepharose were prepared by negative-staining. Panel (b) shows a higher magnification view of panel (a).

To test whether the CCT purified from P-fraction has ATPase activity, ATP was incubated with CCT and analyzed by HPLC using a reversed phase column (Figure 6(a)). Production of ADP from ATP by CCT indicated that the purified CCT has ATPase activity. Intriguingly, CCT was found to possess a GTPase activity (Figure 6(b)). We next investigated whether the presence of GTP influences protease resistance of CCT. As shown in Figure 7 , the purified CCT was completely digested by proteinase $\mathrm{K}$ in the absence of nucleotides. In contrast, CCT was more resistant to protease digestion in the presence of GTP and divalent cations at a level similar to the presence of ATP. Finally, to measure the ATPase activity more exactly, we purified CCT in more large scale using a modified method (supplementary Figures 1, 3, 4), and concentration of free phosphate produced by ATP hydrolysis was determined using Malachite green as an indicator. By this method, the purified CCT showed ATPase activity of $1.21 \mathrm{ATP} / \mathrm{CCT} / \mathrm{min}$. The GTPase activity of CCT was estimated to be $1.24 \mathrm{GTP} / \mathrm{CCT} / \mathrm{mim}$ by the Malachite green method.

\section{DISCUSSION}

In the present study, we established a convenient purification method of CCT. This method uses ATP-Sepharose as a purification step and purified protein had a ring-like complex with a molecular mass of approximately 900 $\mathrm{kDa}$ and contained eight different subunits of approximately $60 \mathrm{kDa}$. The ATP-binding form of CCT was very stable as a complex under various incubation conditions $\left(-30^{\circ} \mathrm{C}\right.$ for 1 week, $37^{\circ} \mathrm{C}$ for $3 \mathrm{~h}$, or $\mathrm{pH} 6.0-8.8$ for $24 \mathrm{~h}$ at $4^{\circ} \mathrm{C}$ ) and freeze drying/thawing (data not shown). In addition, we could purify a smaller CCT complex rich in the $\alpha$ subunit using Heparin-Sepharose. This form of CCT is probably an assembly intermediate or partially disassembled complex as reported by Liou et al. [25]. Recently, it has been shown that ATP hydrolysis of CCT has a dual function in the folding cycle, triggering both lid closure and substrate release into the central chamber [26].

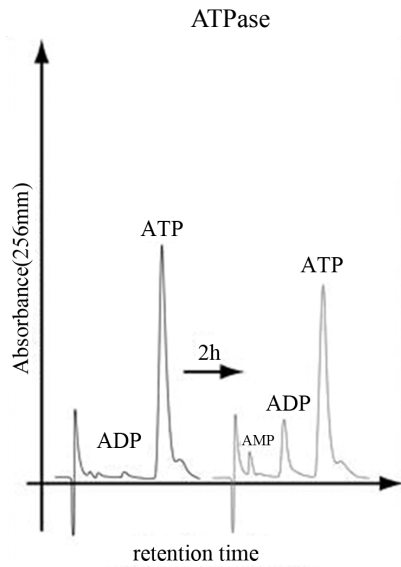

(a)

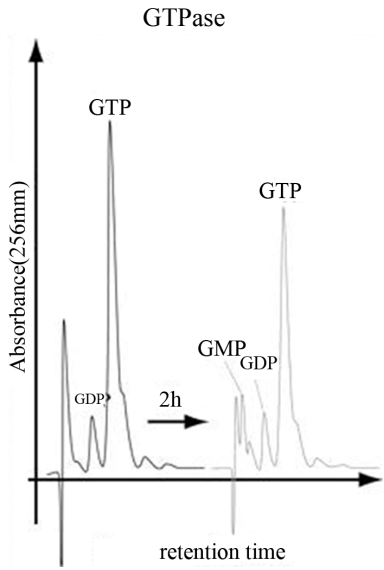

(b)
Figure 6. Nucleotide separation by a $\mathrm{C}_{18}$-reverse phase column. CCT purified from P-fraction was incubated with ATP or GTP at $37^{\circ} \mathrm{C}$ for $2 \mathrm{~h}$. Samples of time 0 and $2 \mathrm{~h}$ were separated by a $\mathrm{C}_{18}$-reverse phase column and absorbance at $256 \mathrm{~nm}$ was recorded.

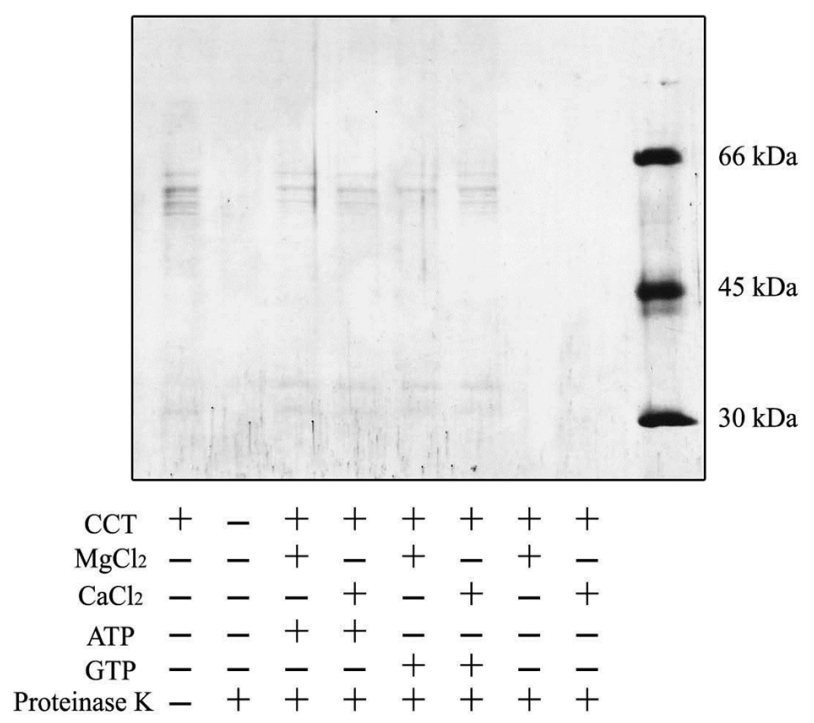

Figure 7. Protease resistance of CCT in the presence of GTP or ATP.CCT purified from P-fraction was incubated and proteinase- $\mathrm{K}$ in the presence or absence of the indicated substances. Samples were analyzed on SDS-PAGE, followed by staining with Coomassie Brilliant Blue.

More importantly, we found in the present study that the CCT purified by ATP-Sepharose possesses a GTPase activity at a level similar to the ATPase activity (1.2 nucleotide/CCT/mim). Recently, GTPase activity of group II chaperonin from thermophilic archaea Thermoplasma acidophilumis was reported [27], and the activity is calculated to be approximately $2.6 \mathrm{GTP} /$ chaperonin/min, although it was lower than ATPase activity (4.6 ATP/chaperonin/min). Thus, the ability to hydrolyze GTP in addition to ATP appears to be common to group 
II chaperonins. We also found that GTP induces a conformational change that provides protease resistance to $\mathrm{CCT}$. It has been reported that CCT is protected from proteinase $\mathrm{K}$ in the presence of ATP and that ATP hydrolysis induce a conformational change from a open form to a closed form [17]. In this process, a built-in lid constructed by helical protrusion is used to close the chaperonin chamber that accommodates unfolded or partially folded substrate proteins. These observations suggest that GTP induces an open-to-close conformational change in the CCT structure. CCT assists in the folding of more than several cytosolic proteins and is essential for tubulin folding in vivo and in vitro [8, 11, 13-15]. As GTP is important for tubulin folding as a structural constituent and affects dynamic instability of tubulin assembly, the GTPase activity of CCT may contribute to directly regulate the tubulin folding in vivo. Thus, the GTPase activity of the CCT may play an important role in the function of CCT in living cells.

\section{ACKNOWLEDGEMENTS}

This work was supported in part by a Grant-in-Aid for Scientific Research to H.I. (Exploratory Research: No. 16651056) from the Japanese Ministry of Education and Science, Sports and Culture.

\section{REFERENCES}

[1] Bukau, B. and Horwich, A.L. (1998) The Hsp70 and Hsp60 chaperone machines. Cell, 14, 351-366. doi:10.1016/S0092-8674(00)80928-9

[2] Hartl, F.U. and Hayer-Hartl, M. (2002) Molecular chaperones in the cytosol: From nascent chain to folded protein. Science, 295, 1852-1858.

doi: $10.1126 /$ science. 1068408

[3] Horwich, A.L., Fenton, W.A., Chapman E. and Farr, G.W. (2007) Two Families of Chaperonin: Physiology and Mechanism. Annual Review of Cell and Developmental Biology, 23, 115-145. doi:10.1146/annurev.cellbio.23.090506.123555

[4] Itoh, H., Kobayashi, R., Wakui, H., Komatsuda, A., Ohtani, H., Miura, A.B., Otaka, M., Masamune, O., Andoh, H., Koyama, K., Sato, Y. and Tashima, Y. (1995) Mammalian 60-kDa stress protein (chaperonin homolog). Identification, biochemical properties, and localization. Journal of Biological Chemistry, 270, 13429-1335.

[5] Itoh H, Komatsuda A, Wakui H, Miura AB, Tashima Y. (1999) Mammalian HSP60 is a major target for an immunosuppressant mizoribine. Journal of Biological Chemistry, 274, 35147-35151. doi:10.1074/jbc.274.49.35147

[6] Itoh H, Komatsuda A, Ohtani H, Wakui H, Imai H, Sawada K, Otaka M, Ogura M, Suzuki A, Hamada F. (2002) Mammalian HSP60 is quickly sorted into the mitochondria under conditions of dehydration. European Journal of Biochemical, 269, 5931-5938. doi:10.1046/j.1432-1033.2002.03317.x

[7] Gutsche, I., Essen, L.O. and Baumeister, W. (1999)
Group II chaperonins: New TRiC(k)s and turns of a protein folding machine. Journal of Molecular Biology, 293, 295-312. doi:10.1006/jmbi.1999.3008

[8] Spiess, C., Meyer, A.S., Reissmann, S. and Frydman, J. (2004) Mechanism of the eukaryotic chaperonin: Protein folding in the chamber of secrets. Trends Cell Biology, 14, 598-604. doi:10.1016/j.tcb.2004.09.015

[9] Sigler, P.B., Xu, Z., Rye, H.S., Burston, S.G., Fenton, W.A. and Horwich, A.L. (1999) Structure and function in GroEL-mediated protein folding. Annual Review of Biochemistry, 67, 581-608. doi:10.1146/annurev.biochem.67.1.581

[10] Iizuka, R., So, S., Inobe, T., Yoshida, T., Zako, T., Kuwajima, K. and Yohda, M. (2004) Role of the helical protrusion in the conformational change and molecular chaperone activity of the archaeal group II chaperonin. Journal of Biology Chemistry, 279, 18834-18839.

[11] Kubota, H. 2002. Function and regulation of cytosolic molecular chaperone CCT. Vitam Horm, 65, 313-331. doi:10.1016/S0083-6729(02)65069-1

[12] Kubota, H., Hynes, G., Carne, A., Ashworth, A. and Willison, K. (1994) Identification of six Tcp-1-related genes encoding divergent subunits of the TCP-1-containing chaperonin. Current Biology, 4, 89-99. doi:10.1016/S0960-9822(94)00024-2

[13] Frydman, J., Nimmesgern, E., Ohtuka, K. and Hartl, F.-U. (1994) Folding of nascent polypeptide chains in a high molecular mass assembly with molecular chaperones. Nature, 370, 111-117. doi:10.1038/370111a0

[14] Tian, G., Vainberg, I.E., Tap, W.D., Lewis, S.A. and Cowan, N.J. (1995) Specificity in chaperonin-mediated protein folding. Nature, 375, 250-253. doi: $10.1038 / 375250 \mathrm{a} 0$

[15] Kubota, S., Kubota, H. and Nagata, K. (2006) Cytosolic chaperonin protects folding intermediates of Gbeta from aggregation by recognizing hydrophobic beta-strands. Proceedings of the National Academy of Sciences, USA, 103, 8360-8365. doi:10.1073/pnas.0600195103

[16] Llorca, O., Martin-Benito, J., Grantham, J., Ritco-Vonsovici, M., Willison, K.R., Carrascosa, J.L. and Valpuesta, J.M. (2001) The 'sequential allosteric ring' mechanism in the eukaryotic chaperonin-assisted folding of actin and tubulin. EMBO Journal, 20, 4065-4075. doi:10.1093/emboj/20.15.4065

[17] Meyer, A.S., Gillespie, J.R., Walther, D., Millet, I.S., Doniach, S. and Frydman, J. (2003) Closing the folding chamber of the eukaryotic chaperonin requires the transition state of ATP hydrolysis. Cell, 113, 369-381. doi:10.1016/S0092-8674(03)00307-6

[18] Lin, P. and Sherman, F. (1997) The unique hetero-oligomeric nature of the subunits in the catalytic cooperativity of the yeast Cct chaperonin complex. Proceedings of the National Academy of Sciences, USA, 94, 10780-10785. doi:10.1073/pnas.94.20.10780

[19] Rivenzon-Segal, D., Wolf, S.G., Shimon, L., Willison. K.R. and Horovitz, A. (2005) Sequential ATP-induced allosteric transitions of the cytoplasmic chaperonin containing TCP-1 revealed by EM analysis. Nature Structural and Molecular Biologlogy, 12, 233-237. doi:10.1038/nsmb901

[20] Laemmli, U.K. (1970) Cleavage of structural proteins during the assembly of the head of bacteriophage T4. 
Nature, 227, 680-685. doi:10.1038/227680a0

[21] Towbin, H., Staehelin, T. and Gordon, J. (1979) Electrophoretic transfer of proteins from polyacrylamide gels to nitrocellulose sheets: Procedure and some applications. Proceedings of the National Academy of Sciences, USA, 76, 4350-4354. doi:10.1073/pnas.76.9.4350

[22] O'Farrell, P.H. (1975) High resolution two-dimensional electrophoresis of proteins. Journal of Biological Chemistry, 250, 4007-4021.

[23] Martin, B., Pallen, C.J., Wang, J.H. and Graves, D.J. (1985) Use of fluorinated tyrosine phosphates to probe the substrate specificity of the low molecular weight phosphatase activity of calcineurin. Journal of Biological Chemistry, 260, 14932-14937.

[24] Lake, J.A. (1978) Electron microscopy of specific proteins: Three-dimensional mapping of ribosomal proteins using antibody labels. In: Koehler, J.K., Eds., Advanced techniques in biological electron microscopy, Springer-Verlag, New York, 2, 173-211.

[25] Liou, A.K. and Willison, K.R. (1997) Elucidation of the subunit orientation in CCT (chaperonin containing TCP1) from the subunit composition of CCT micro-complexes. EMBO Journal, 16, 4311-4316. doi:10.1093/emboj/16.14.4311

[26] Douglas, N.R., Reissmann, S., Zhang, J., Chen, B., Jakana, J., Kumar, R., Chiu, W. and Frydman, J. (2011) Dual action of ATP hydrolysis couples lid closure to substrate release into the group II chaperonin chamber. Cell, 144, 240-252. doi:10.1016/j.cell.2010.12.017

[27] Hirai, H., Noi, K., Hongo, K., Mizobata, T. and Kawata, Y. (2008) Functional characterization of the recombinant group II chaperonin alpha from thermoplasma acidophilum. Journal of Biochemistry, 143, 505-515. doi:10.1093/jb/mvm241

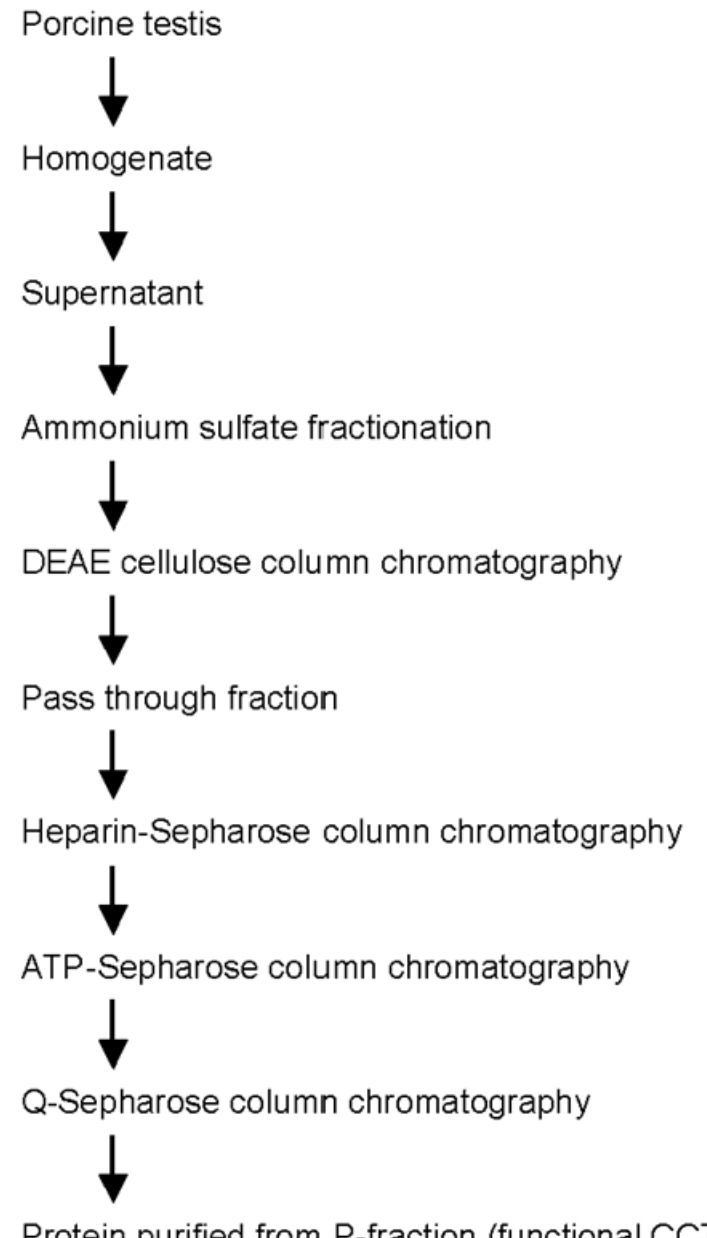

Protein purified from $\mathrm{P}$-fraction (functional CCT)

Supplementary Figure 1. Flow chart of CCT purification in large scale. 
$\underline{\text { Small scale purification }}$

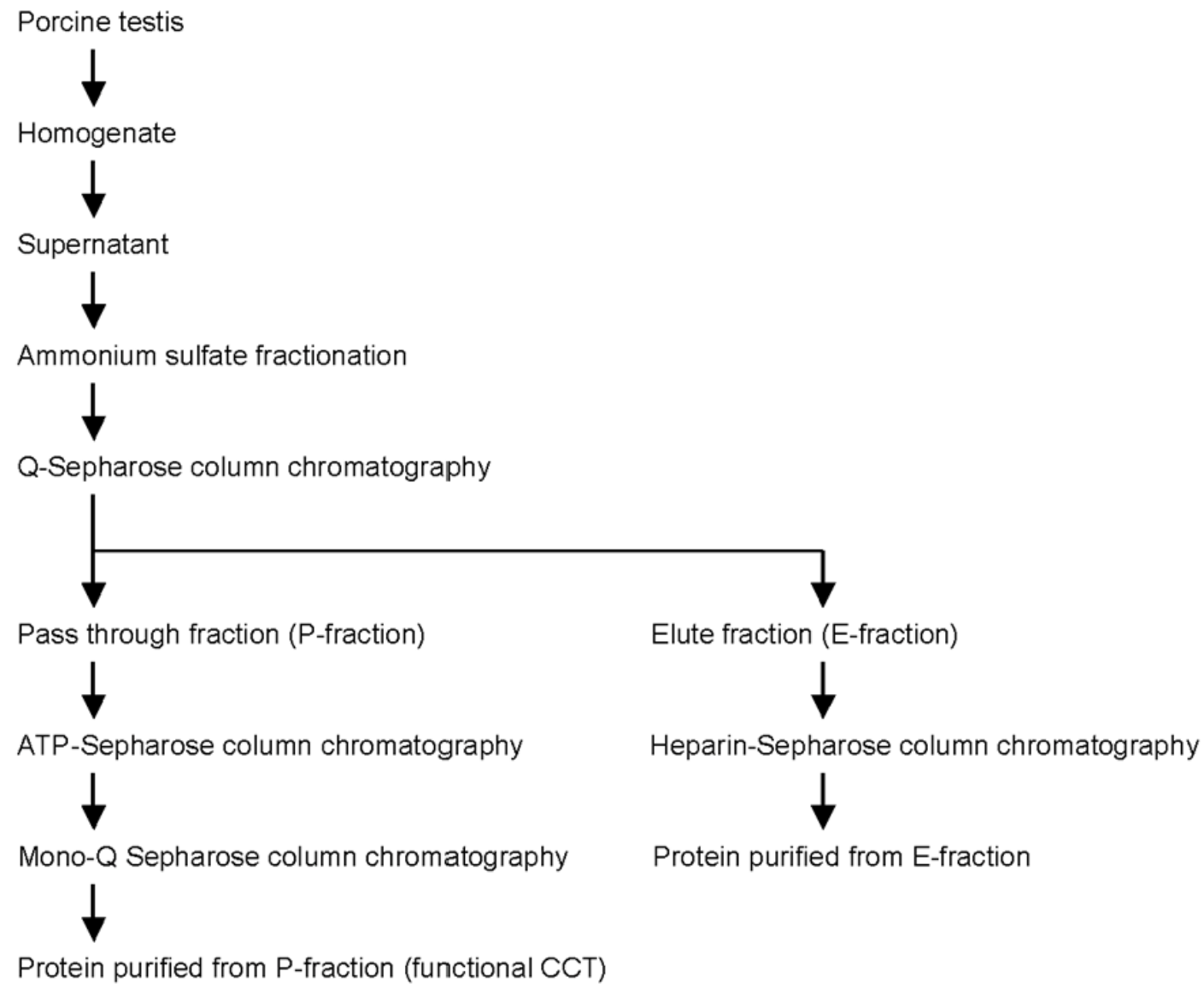

Supplementary Figure 2. Flow chart of CCT purification in small scale.

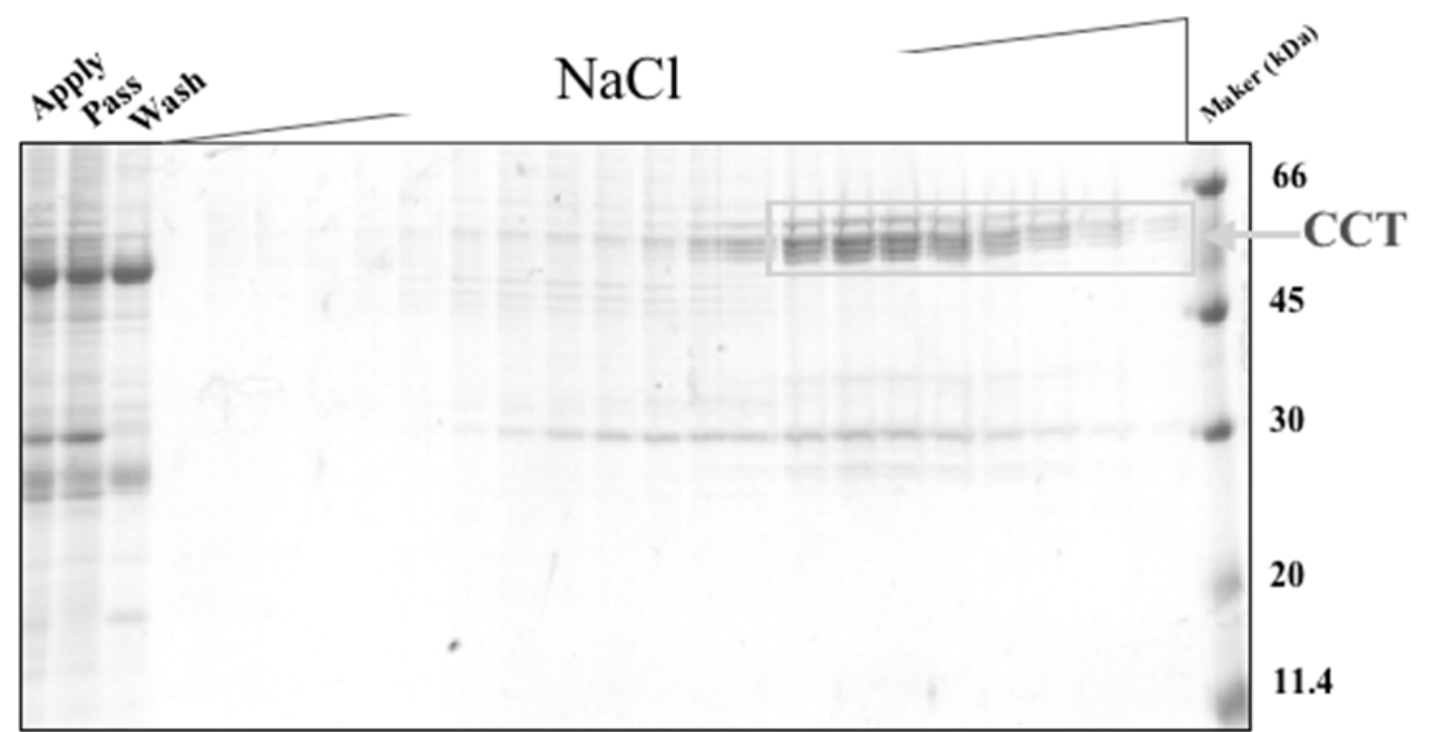

Supplementary Figure 3. SDS-PAGE of fractions eluted from the heparin-Sepharose column for large scale purification (see Supplementary Figure 1). 


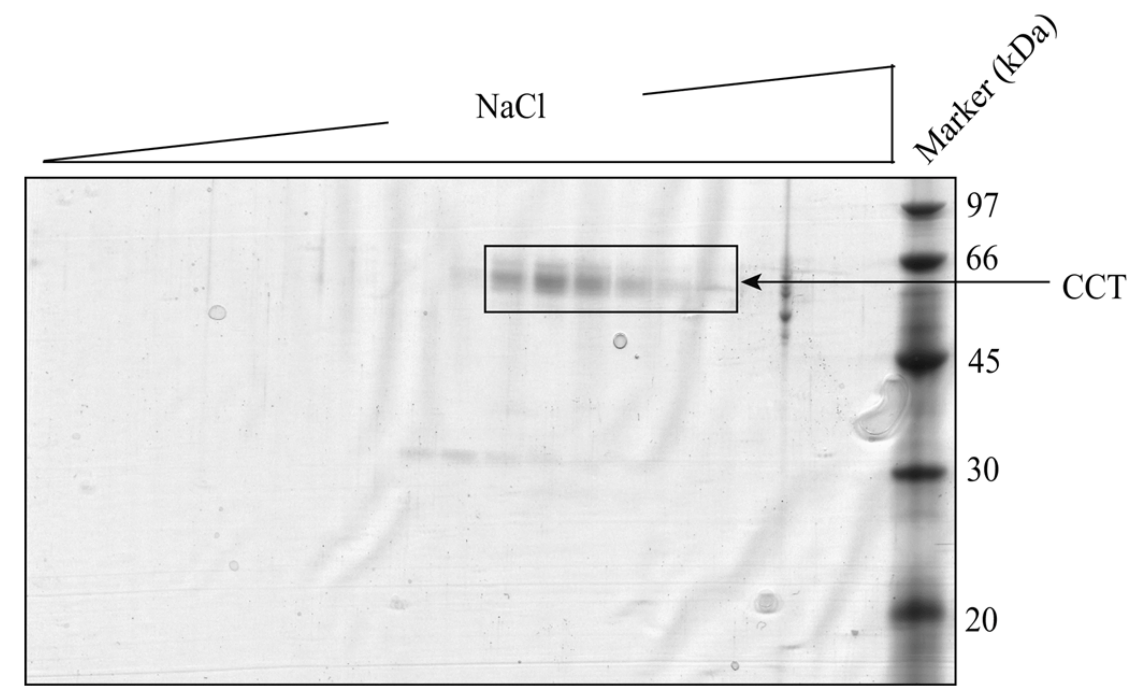

Supplementary Figure 4. SDS-PAGE of fractions eluted from the Q-Sepharose column for large scale purification (see Supplementary Figure 1).

Abbreviations: CCT, chaperonin containing t-complex polypeptide 1; HSP60, 60-kDa heat shock protein. 